\title{
Investigation of second harmonic generation and multispectral imaging as new contrast mechanisms in scanning laser optical tomography
}

Lena Nolte, Georgios C. Antonopoulos, Alexander Heisterkamp, Tammo Ripken, Heiko Meyer

Lena Nolte, Georgios C. Antonopoulos, Alexander Heisterkamp, Tammo Ripken, Heiko Meyer, "Investigation of second harmonic generation and multispectral imaging as new contrast mechanisms in scanning laser optical tomography ," Proc. SPIE 10499, Three-Dimensional and Multidimensional Microscopy: Image Acquisition and Processing XXV, 1049913 (23 February 2018); doi: 10.1117/12.2289509

SPIE. Event: SPIE BiOS, 2018, San Francisco, California, United States 


\title{
Investigation of second harmonic generation and multispectral imaging as new contrast mechanisms in scanning laser optical tomography
}

\author{
Lena Nolte $^{* a}$, Georgios C. Antonopoulos ${ }^{\mathrm{a}}$, Alexander Heisterkamp ${ }^{\mathrm{b}}$, Tammo Ripken $^{\mathrm{a}}$, Heiko Meyer $^{\mathrm{a}}$ \\ ${ }^{a}$ Industrial and Biomedical Optics Department, Laser Zentrum Hannover e.V., Hollerithallee 8, \\ 30419 Hannover , Germany; ${ }^{b}$ Institut für Quantenoptik, Leibniz Universität Hannover, Hannover, \\ Germany
}

\begin{abstract}
Scanning laser optical tomography (SLOT) is a 3D imaging technique, based on the principle of computed tomography to visualize samples up to magnitude of several centimeters. Intrinsic contrast mechanisms as absorption, scattering and autofluorescence provide information about the 3D architecture and composition of the sample.

Another valuable intrinsic contrast mechanism is second harmonic generation (SHG), which is generated in noncentrosymmetric materials and commonly used to image collagen in biological samples. The angular dependence of the SHG signal, however, produces artifacts in reconstructed optical tomography datasets (OPT, SLOT). Thus, successful use of this intrinsic contrast mechanism is impaired. We investigate these artifacts by simulation and experiment and propose an elimination procedure that enables successful reconstruction of SHG-SLOT data.

Nevertheless, in many cases specific labeling of certain structures is necessary to make them visible. Using multiple dyes in one sample can lead to crosstalk between the different channels and reduce contrast of the images. Also autofluorescence of the sample itself can account for that. By using multispectral imaging in combination with spectral unmixing techniques, this loss can be compensated. Therefore either a spectrally resolved detection path, or spectrally resolved excitation is required. Therefore we integrated a white supercontinuum light source in our SLOT-setup that enables a spectral selection of the excitation beam and extended the detection path to a four channel setup. This enables the detection of three fluorescence channels and one absorption channel in parallel, and increases the contrast in the reconstructed 3D images significantly.
\end{abstract}

Keywords: Scanning Laser Optical Tomography, 3D Microscopy, Tomographic imaging, Nonlinear Optics, Second Harmonic Generation, Multispectral imaging, Spectral unmixing

\section{INTRODUCTION}

Three dimensional imaging is of high interest to understand the morphology and development of biological samples. There are many imaging techniques available, mainly differing in field of view, resolution and contrast mechanisms. One common technique for in toto imaging of mesoscopic samples is micro-computed tomography. This method uses the attenuation of x-rays by the sample as a contrast mechanism and is mainly applied to bony structures ${ }^{1}$. For more specific visualization of soft tissue, light based techniques as confocal microscopy are used. Using the specific labeling of a certain target structure in combination with fluorescence microscopy provides high contrast and high resolution images in 3D. Since many fluorophores overlap spectrally, which results in low contrast images, multispectral imaging in combination with spectral unmixing is widely used. This can also eliminate the background signal that is due to autofluorescence ${ }^{2}$. There are unmixing algorithms available that require some a priori knowledge about the sample as non-negative least squares and blind algorithms as nonnegative matrix factorization ${ }^{3}$ or spectral phasor analysis ${ }^{4}$.

To increase penetration depth and reduce photo damage, excitation light in the near infrared can be used resulting in images based on two photon fluorescence or second harmonic generation (SHG). Non centrosymmetric structures, as collagen and myosin, can be visualized in 3D using SHG without the need of an external stain. This has been used in several studies to visualize cornea ${ }^{5}$, atherosclerotic arterial tissue ${ }^{6}$, skin $^{7,8}$ or cancer ${ }^{9-12}$. Both methods, confocal

*l.nolte@lzh.de; phone 0049511 2788-386; fax 0049511 2788-100; www.lzh.de

Three-Dimensional and Multidimensional Microscopy: Image Acquisition and Processing XXV, edited by

Thomas G. Brown, Carol J. Cogswell, Tony Wilson, Proc. of SPIE Vol. 10499, 1049913

(C) 2018 SPIE · CCC code: 1605-7422/18/\$18 · doi: 10.1117/12.2289509 
microscopy and multiphoton microscopy, provide high resolution images, but suffer from a small field of view. In combination with mosaicking ${ }^{13}$, the imaging depth is limited by the working distance of the objective. To enlarge the field of view, an optical method based on the principle of computed tomography, has been shown for the first time by Brown et al. ${ }^{14}$. This method was named by Sharpe in 2002 Optical Projection Tomography (OPT) $)^{15}$ and uses absorption and scattering as contrast mechanisms. Projection images are generated of a sample from different angles and reconstructed in 3D using the inverse Radon transform. To further increase the collection efficiency, SLOT was developed in $2011^{16}$. SLOT uses absorption, scattering and fluorescence as contrast mechanism, that can be provided by the sample intrinsically ${ }^{17,18}$ or using a specific labeling ${ }^{19}$. Also the surface of non-transparent samples can be visualized in $3 \mathrm{D}^{20}$.

To increase the contrast and the applicability of SLOT to a larger variety of samples and fluorophores, it would be valuable to apply multispectral imaging and linear unmixing. Therefore, we extended the SLOT setup by two additional channels that enable the simultaneous acquisition of three fluorescence/scattering channels in addition to one absorption channel. Additionally the $\mathrm{cw}$-light source was replaced by a supercontinuum light source that enables continuous tuning of the excitation wavelength from $400-750 \mathrm{~nm}$.

Furthermore, a fs-pulsed laser in the near infrared is integrated providing the possibility to generate a second harmonic signal (SHG). The reconstruction of SHG-SLOT data is, however, challenged by the requirement of sample rotation. Due to the angle dependence of SHG, the SHG signal of individual scatterer is not always constant during rotation, as it is required by the inverse Radon transform. In this paper we investigated this behavior by simulation and experiment and propose a solution for the usage of SHG-SLOT.

\section{THEORY}

\subsection{Scanning Laser Optical Tomography}

The principle of scanning laser optical tomography (SLOT) is equivalent to the principle of computed tomography. In contrast to $\mathrm{x}$-rays, SLOT uses light in the visible or near infrared range to generate contrast inside the sample. The light of three laser diodes $(450 \mathrm{~nm} / 520 \mathrm{~nm} / 635 \mathrm{~nm})$ is coupled into a single mode fiber to generate a TEM 00 beam profile (see Figure 1). The diameter of this laser beam can be adjusted in diameter by a zoom telescope. This enables the adjustment of the numerical aperture on the size of the sample. Using two scanning mirrors, that are positioned in the back focal plane of an imaging lens, the light is focused onto the sample, which is connected to a rotation motor. The transmitted light is subsequently collected and directed on a photo diode, to measure the attenuation of the sample. Simultaneously, scattered or fluorescent light (depending on the used filter) can be detected orthogonally to the optical axis by a photo multiplier tube (PMT). The beam is scanned in a meander pattern over the sample, to generate a two dimensional projection image of the sample. This two dimensional projection can be described by the Radon transform. Subsequently, the sample is rotated by a small angle $\varphi$ and a projection image is acquired again. This procedure is repeated until a full revolution of the sample is achieved. The set of Radon transforms of one single plane for multiple angles is also called sinogram.

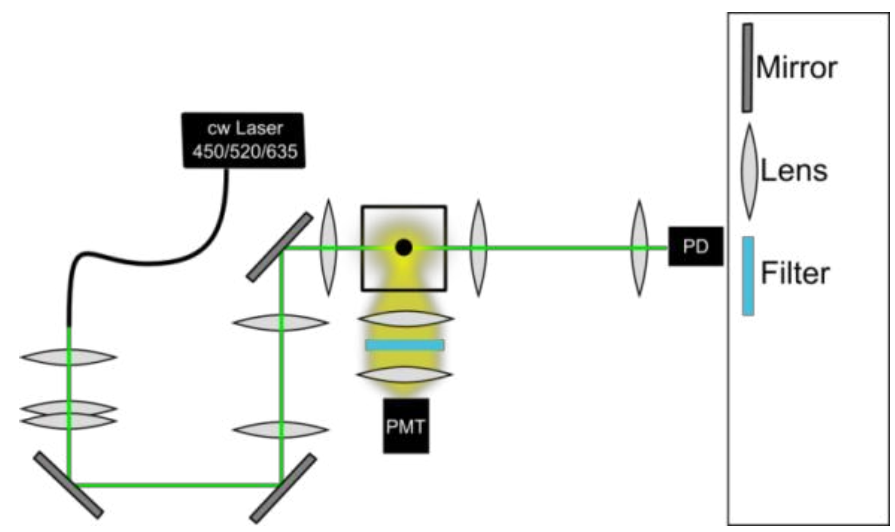

Figure 1. Setup of a Scanning Laser Optical Tomograph (SLOT). The light of three laser diodes (450nm, $520 \mathrm{~nm}, 635 \mathrm{~nm})$ is coupled into a fiber and subsequently collimated. The beam diameter can be varied by a zoom telescope to adjust the numerical aperture of the imaging setup. Two scanning mirrors enable the two dimensional scanning of the beam over the sample. Simultaneously the extinction of the sample can be measured in transmission direction and fluorescence or scattering orthogonal to the optical axis. 
Using this information the 2D composition of one single plane can be reconstructed by the invers Radon transform. One important condition for the successful reconstruction is that the absorbance, scatter- or fluorescence intensity stays constant during the full revolution of the sample. Otherwise, the inverse Radon transform cannot be applied sufficiently.

\subsection{Second Harmonic Generation}

The second harmonic generation (SHG) intensity depends strongly on the orientation of the laser polarization and the orientation of the sample. Hence, a reference coordinate system is defined in Figure 1. The electromagnetic field $\vec{E}$ is positioned in the $x-y$-plane und tilted by the polarization angle $\alpha$ to the $x$-axis. The sample and the sample coordinate system (cx, cy, cz) is tilted by the tilt angle $\theta$ to the $x$-axis and by the rotation angle $\varphi$ to the $y$-axis. Sample rotation occurs around the $\mathrm{x}$-axis and is equivalent to a rotation by $\varphi$.
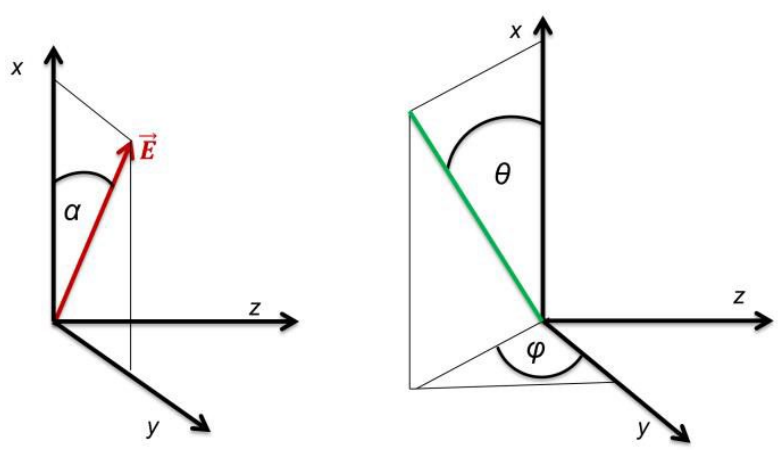

Figure 2. Reference coordinate system. The linear polarization of the incident light (red arrow) is tilted by the polarization angle $\alpha$ to the $\mathrm{x}$-axis. Light propagates in $\mathrm{z}$-direction. The collagen fiber (green rod) is tilted by the tilt angel $\theta$ to the $\mathrm{x}$-axis and by the rotation angle $\varphi$ to the $y$-axis. Sample rotation for a SLOT measurement occurs around the $\mathrm{x}$-axis. The sample coordinate system is indicated by cx, cy, cz.

The generation of a SHG signal depends also on the sample itself. Only materials with a non-zero second order susceptibility tensor $X_{i j k}^{(2)}$ are able to generate a SHG signal. This is only the case for noncentrosymmetric materials. One biological non centrosymmetric material is collagen. There are some common symmetry assumptions for collagen to reduce the number of independent tensor coefficients like intrinsic permutation symmetry ${ }^{21}$, cylindrical symmetry ${ }^{22}$ and Kleinmann symmetry ${ }^{23}$. Using these simplifications, the induced SHG polarization $\vec{P}_{S H G}$ can be calculated by equation 1 , where $\varepsilon_{0}$ is the vacuum permittivity and $d_{i s}$ the contracted description of the susceptibility components ${ }^{\mathrm{b}}$ :

$$
\vec{P}_{S H G}=\varepsilon_{0} \bullet\left[\begin{array}{cccccc}
d_{11} & d_{12} & d_{12} & 0 & 0 & 0 \\
0 & 0 & 0 & 0 & 0 & d_{12} \\
0 & 0 & 0 & 0 & d_{12} & 0
\end{array}\right] \bullet\left[\begin{array}{c}
E_{c x}^{2} \\
E_{c y}^{2} \\
E_{c z}^{2} \\
2 E_{c y} E_{c z} \\
2 E_{c x} E_{c z} \\
2 E_{c x} E_{c y}
\end{array}\right] .
$$

The components of the electromagnetic field are here given for the coordinate system of the sample (cx, cy, cz). To transform the incident electromagnetic field $\vec{E}$ in the coordinate system of the sample (cx, cy, cz) it needs to be multiplied by the rotation matrix $\underline{M}=\underline{R}_{y}(\theta) \times \underline{R}_{x}(\varphi)$, which produces a rotation by $\theta$ around the $\mathrm{y}$-axis and a rotation by $\varphi$ around the $x$-axis resulting in:

\footnotetext{
${ }^{b}$ The index s combines the value of $\mathrm{j}$ and $\mathrm{k}$ by:
}

\begin{tabular}{c|c|c|c|c|c|c}
$\mathbf{s}$ & 1 & 2 & 3 & 4 & 5 & 6 \\
\hline $\mathbf{j}$ & 1 & 2 & 3 & 2 & 3 & 1 \\
\hline $\mathbf{k}$ & 1 & 2 & 3 & 3 & 1 & 2
\end{tabular}




$$
\vec{E}_{C}=\underline{M} \cdot \vec{E}=\left[\begin{array}{ccc}
\cos (\theta) & \sin (\theta) \sin (\varphi) & -\sin (\theta) \cos (\varphi) \\
0 & \cos (\varphi) & \sin (\varphi) \\
\sin (\theta) & -\cos (\theta) \sin (\varphi) & \cos (\theta) \cos (\varphi)
\end{array}\right] \cdot\left[\begin{array}{c}
E_{0} \cos (\alpha) \\
E_{0} \sin (\alpha) \\
0
\end{array}\right] .
$$

The generated SHG intensity is now proportional to $\left|\vec{P}_{S H G}\right|^{2}$.

\subsection{Multispectral Imaging}

The emission spectra of fluorophores show a broad profile, which results in spectral overlap (see Figure 7). This overlap cannot always be excluded by a sophisticated choice of emission filters. Especially if fluorescent proteins are required, fluorophore options are limited and crosstalk is a common challenge.

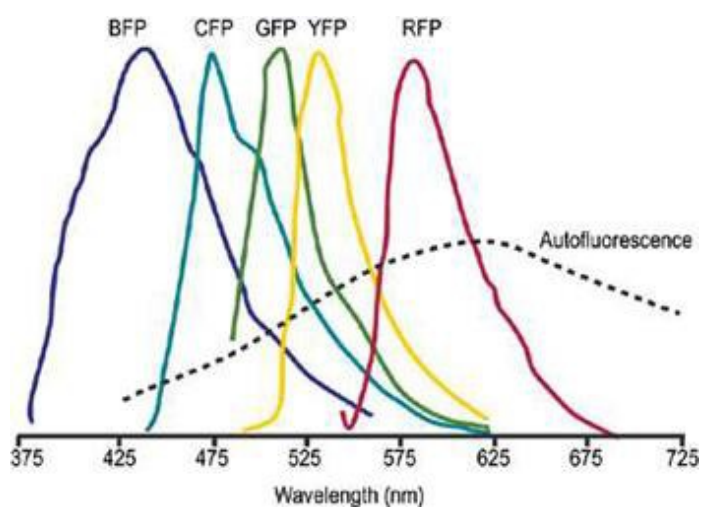

Figure 3. Schematic drawing of emission spectra from fluorescent proteins and a typical autofluorescence curve ${ }^{24}$.

However, by detecting multiple acquisition channels, unmixing algorithms can be used to separate the fluorophores more efficient. There is a variety of unmixing algorithms available. In this paper we used the non-negative matrix factorization (NMF) plugin for Image ${ }^{3,25}$.

\section{SETUP MODIFICATION}

To adjust the SLOT setup for the generation and detection of a SHG signal, two major changes need to be applied to the setup. First, a fs-pulsed light source in the near infrared was integrated, to enable a high photon density inside the sample. Therefore, the light of a Chameleon Ultra II (Coherent, Inc.) is overplayed with the cw-laser (see Figure 4). The beam is adjustable in intensity, using a half-wave plate and a polarizing beam cube and in diameter by a zoom-telescope. Second, the detection path needed to be modified. Since a SHG signal is mainly generated in forward direction, SHG detection will also occur in forward direction. Therefore, a second photo multiplier tube is positioned in forward direction in combination with two filters, to block remaining transmitted laser light. To maintain the possibility to measurer the transmission of the sample, a dichroic mirror is additionally placed in the forward detection path that directs the laser light on the photodiode. For multispectral imaging, additionally, a third PMT is integrated in the setup, to collect fluorescence or scattered light from the top side, orthogonally to the optical axis. This way, three fluorescence/scattering channels can be acquired simultaneously in addition to the absorption measurement by the photodiode. Also the cw-laser diodes were replaced by a supercontinuum light source (Rock 400, Leukos), that is connected to a filter unit. This way the excitation wavelength can be adjusted specifically to the requirements of the sample. Furthermore, the excitation wavelength can be tuned continuously from 400-750 nm to measure the wavelength dependent extinction, scattering or fluorescence of the sample. 


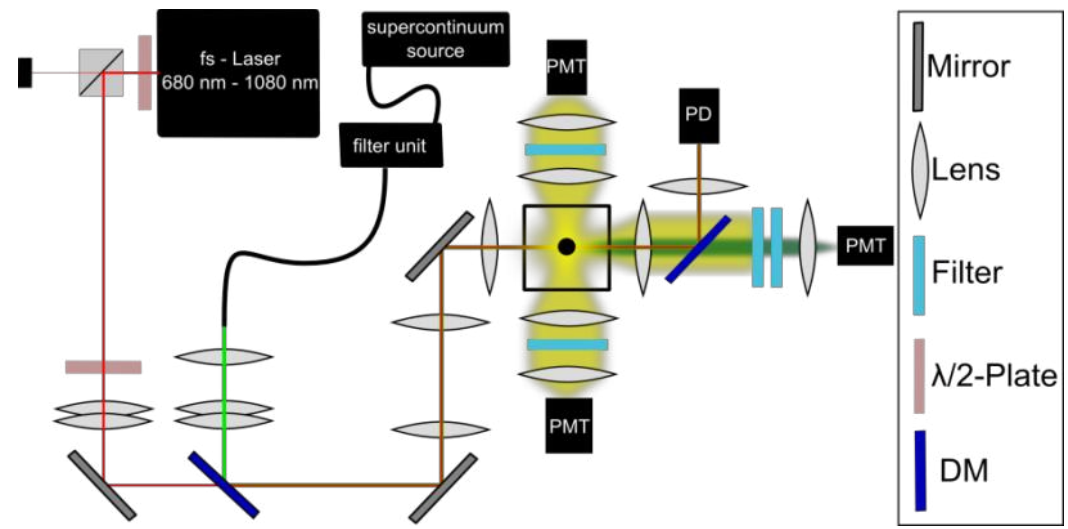

Figure 4. Modification of the SLOT setup. The cw-laser diodes where replaced by a supercontinuum source, that is connected to a filter unit. Additionally, a fs-pulsed laser is integrated, that is capable to generate a SHG signal. Both beams are overplayed and directed on the sample. In total three PMTs are integrated into the detection channel, two orthogonally to the optical axis and one in transmission direction. Furthermore, a dichroic mirror is positioned in forward direction that directs the laser light onto a photodiode.

\section{SHG-SLOT}

\subsection{Numerical Simulations}

Using equation 1 and 2, the squared SHG polarization $\left|\vec{P}_{S H G}\right|^{2}$ can be calculated numerically. The ratio $\mathrm{d}_{11} / \mathrm{d}_{12}$ is assumed to be $1.3^{26}$. For simplification, the following calculations are performed with $\mathrm{d}_{11}=1$. The squared SHG polarization can be calculated for any combination of the polarization angle $\alpha$, the tilt angle $\theta$ and the rotation angle $\varphi$. Figure 5 shows two simulations of the SHG intensity for all values of $\varphi$ form $0^{\circ}$ to $360^{\circ}$ (left: $\alpha=0^{\circ}$ and $\theta=45^{\circ}$; right: $\alpha=90^{\circ}$ and $\left.\theta=45^{\circ}\right)$.
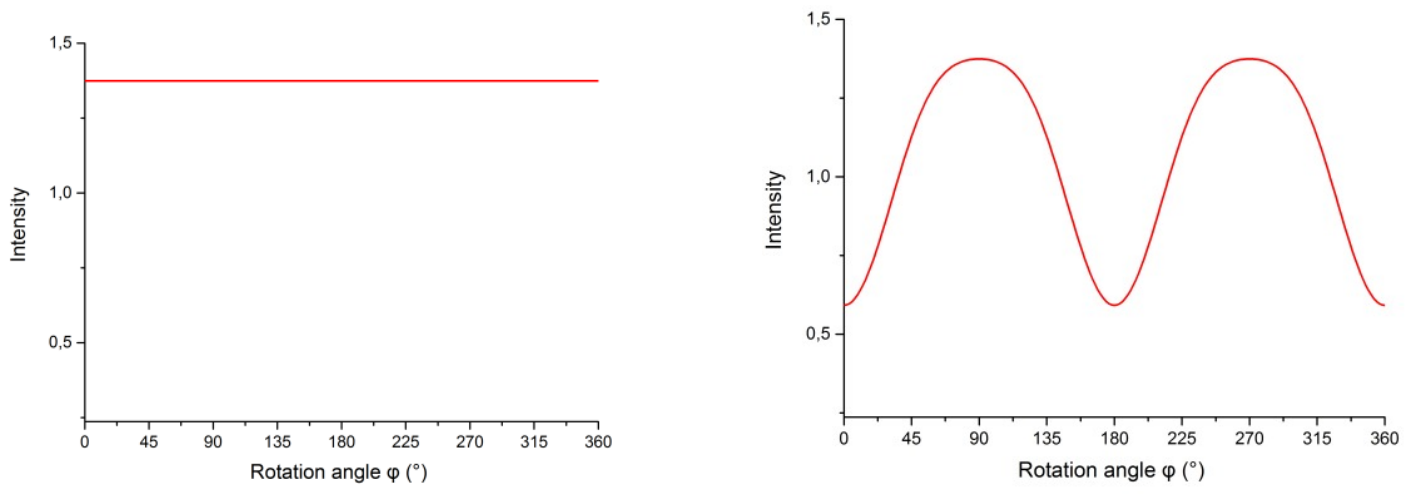

Figure 5. Numerical simulation of the SHG intensity for a full revolution of the sample $\left(\varphi=0-360^{\circ}\right)$. The left side shows the simulation for $\alpha=0^{\circ}$ and $\theta=45^{\circ}$ and the right side for $\alpha=90^{\circ}$ and $\theta=45^{\circ}$.

Only on the left side, where $\alpha$ is set to $0^{\circ}$, the SHG intensity stays constant during a full revolution of the sample. This is not obvious, since for any other polarization angle the SHG intensity varies during sample rotation. Due to the nature of the inverse Radon transform, only the first case can be reconstructed successfully.

\subsection{Experimental Validation}

The behavior that was predicted in the section above was also analyzed experimentally. Therefore, a fascicle from a rat tail tendon was optically cleared in benzyl benzoate and measured using the setup described in section 3. In Figure 6 the measured SHG intensity is displayed for a full revolution of the fascicle using an input polarization at $\alpha=0^{\circ}$ (orange) 
and $\alpha=90^{\circ}$ (green). As simulated before, the SHG intensity fluctuates strongly for $\alpha=90^{\circ}$ and is almost constant for $\alpha=0^{\circ}$. Using this imaging condition, SHG-SLOT data can now be reconstructed successfully.

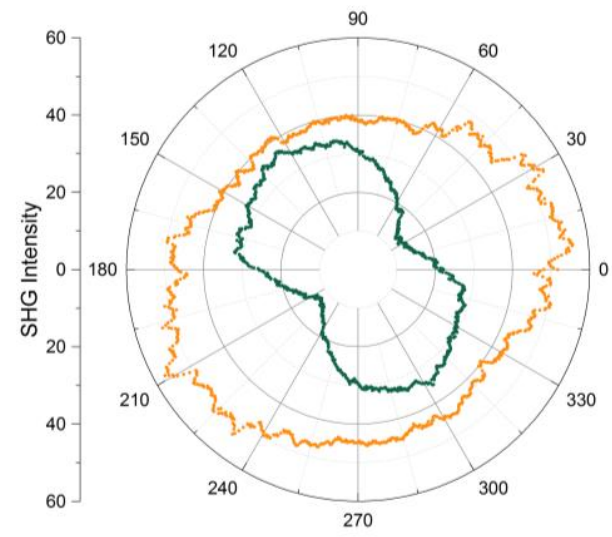

Figure 6. Intensity measurements on a rat tail tendon for a full sample revolution using diffent input polarizations (orange: $\alpha=0^{\circ}$ and green: $\alpha=90^{\circ}$ ).

\section{MULTISPECTRAL-SLOT}

Using the new SLOT setup, there is a variety of unmixing procedures available. The most common application for spectral unmixing is the separation of two or more fluorophores after simultaneous detection of multiple detection channels. Therefor we imaged a sample that contains fluorescent beads labeled either with Lucifer yellow or Fluorescein (see Figure 7 left). This sample was imaged using the three PMT channels in two rounds using different fluorescence filters: 510/10 BP, 525/26 BP, 556/24 BP, 589/15 BP, 620/60 BP and 679/41 BP. Here we show the successful separation of fluorescent beads, that are labeled either with Lucifer yellow or Fluorescein. The acquired images for 510/10 BP and 620/60 BP are shown in Figure 7 A - C. Due to the strong spectral overlap of both fluorophores, the channels cannot be separated. Figure 7 D - E shows the channels after spectral unmixing using the non-negative matrix factorization. Now both fluorophores can be displayed separately.
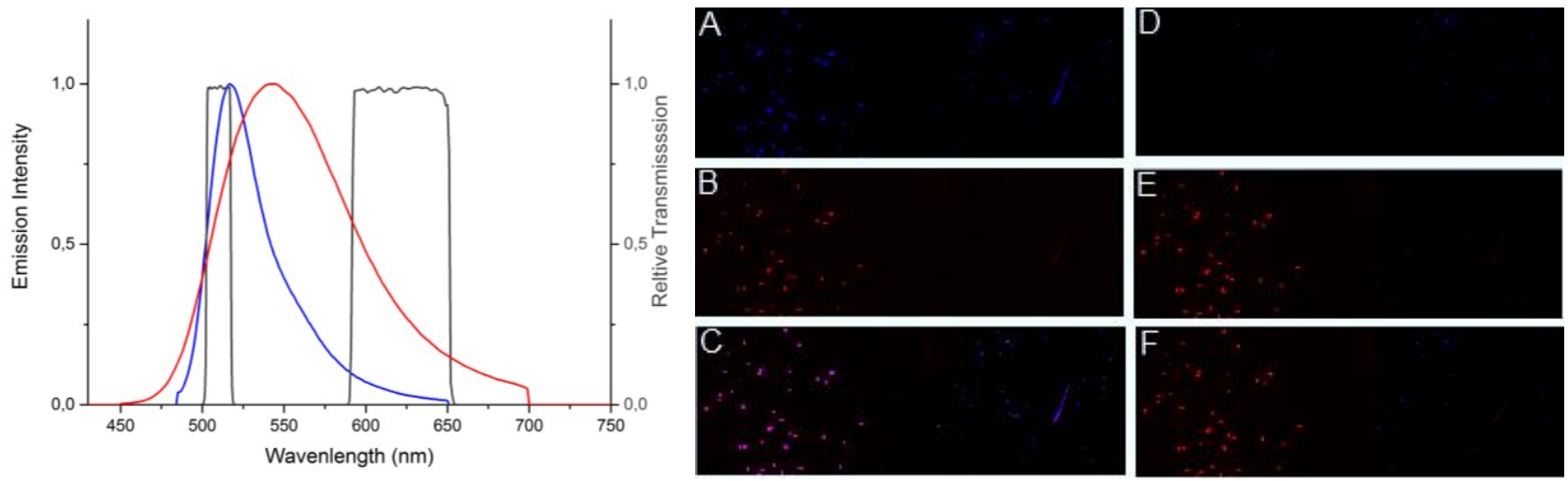

Figure 7. Left: Emission Spectra of Lucifer yellow (red) and Fluorescein (blue) are shown with the transmission curves of two filters (510/10 BP and 620/60 BP). Right: Fluorescent beads, filled with Lucifer yellow (left in each image) and Fluorecein (right in each image) were visualized using the new SLOT setup. (A) Fluorecence image using a 510/10 BP. The fluorescence of both fluophores is visible. (B) Fluorecence image using a 620/60 BP. Mainly the fluorescence of Lucifer yellow is visible. (C) Overlay of A and B. Lucifer Yellow appears in in both channels and apears due to the overly magenta. (D) Fluorecein channel after spectral unmixing. (E) Lucifer yellow channel after spectral unmixing. (F) Overlay of D and E. The contrast between both flurophore is improved. 


\section{CONCLUSION}

In this paper we showed the successful reconstruction of SHG-SLOT data. By choosing a laser polarization that is aligned parallel to the axis of rotation intensity fluctuations during sample rotation can be eliminated, despite the angular dependence of SHG. This enables the successful reconstruction of the data using the inverse Radon transform. Additionally, multispectral imaging was used to enhance the contrast of strongly overlapping fluorophores. This enables a more flexible choice of fluorophores inside the sample without the loss of contrast. But not only the emission of fluorophores can be used for unmixing. The newly integrated supercontinuum source enables a very versatile choice of the excitation light. Due to differing excitation spectra, unmixing of fluorophores can also be improved. But also other contrast mechanisms as scattering or absorption can now be unmixed. The attenuation or scattering of non-fluorescent dyes can be measured for different wavelength. In total, all these extensions of the setup improve the applicability of SLOT. In case of bad contrast between different fluorophores or in respect to sample background, spectral unmixing can be applied to all the detection channels: absorption, scattering and fluorescence. Furthermore, SHG can be used as a new contrast to visualize non centrosymmetric structures, as collagen, inside the sample.

\section{ACKNOWLEDGEMENTS}

This work was supported by the German Federal Ministry of Education and Research (Pionieraxon: 031L0062B) and by the Ministry of Lower Saxony and the VolkswagenStiftung (both BIOFABRICATION FOR NIFE: VWZN2860, http://biofabrication.info/).

\section{REFERENCES}

[1] Boerckel, J.D., Mason, D.E., McDermott, A.M., and Alsberg, E., "Microcomputed tomography: Approaches and applications in bioengineering," Stem Cell Research and Therapy 5(6), 1-12 (2014).

[2] Zimmermann, T., "Spectral imaging and linear unmixing in light microscopy," Advances in Biochemical Engineering/Biotechnology 95, 245-265 (2005).

[3] Neher, R.A., Mitkovski, M., Kirchhoff, F., Neher, E., Theis, F.J., and Zeug, A., "Blind source separation techniques for the decomposition of multiply labeled fluorescence images," Biophysical Journal 96(9), 37913800 (2009).

[4] Fereidouni, F., Bader, A.N., and Gerritsen, H.C., "Spectral phasor analysis allows rapid and reliable unmixing of fluorescence microscopy spectral images," Optics Express 20(12), 12729 (2012).

[5] Matteini, P., Ratto, F., Rossi, F., Cicchi, R., Stringari, C., Kapsokalyvas, D., Pavone, F.S., and Pini, R., "Photothermally-induced disordered patterns of corneal collagen revealed by SHG imaging.," Optics express 17(6), 4868-78 (2009).

[6] Cicchi, R., Baria, E., Matthäus, C., Lange, M., Lattermann, A., Brehm, B.R., Popp, J., and Pavone, F.S., "Characterization of atherosclerotic arterial tissue using combined SHG and FLIM microscopy," in Adv. Microsc. Tech. IV; Neurophotonics II, 95360N (2015).

[7] Cicchi, R., Kapsokalyvas, D., De Giorgi, V., Maio, V., Van Wiechen, A., Massi, D., Lotti, T., and Pavone, F.S., "Scoring of collagen organization in healthy and diseased human dermis by multiphoton microscopy," Journal of Biophotonics 3(1-2), 34-43 (2009).

[8] Yasui, T., Tohno, Y., and Araki, T., "Characterization of collagen orientation in human dermis by twodimensional second-harmonic-generation polarimetry,” Journal of Biomedical Optics 9(2), 259 (2004).

[9] Waters, J.C., and Wittmann, T., [Quantitative imaging in cell biology], Academic Press (2014).

[10] Brown, E., McKee, T., diTomaso, E., Pluen, A., Seed, B., Boucher, Y., and Jain, R.K., "Dynamic imaging of collagen and its modulation in tumors in vivo using second-harmonic generation," Nature Medicine 9(6), 796801 (2003).

[11] Lin, S.-J., Jee, S.-H., Kuo, C.-J., Wu, R.-J., Lin, W.-C., Chen, J.-S., Liao, Y.-H., Hsu, C.-J., Tsai, T.-F., et al., "Discrimination of basal cell carcinoma from normal dermal stroma by quantitative multiphoton imaging.," Optics letters 31(18), 2756-8 (2006).

[12] Provenzano, P.P., Eliceiri, K.W., Campbell, J.M., Inman, D.R., White, J.G., and Keely, P.J., “Collagen 
reorganization at the tumor-stromal interface facilitates local invasion," BMC Medicine 4(1), 38 (2006).

[13] Gareau, D.S., Patl, Y.G., Aranda, I., Li, Y., Halpern, A.C., Nehal, K.S., and Rajadhyaksha, M., "Confocal mosaicing microscopy in skin excisions: a demonstration of rapid surgical pathology," Journal of Microscopy 49(18), 1841-1850 (2009).

[14] Brown, C.S., Burns, D.H., Spelman, F.A., and Nelson, A.C., "Computed tomography from optical projections for three-dimensional reconstruction of thick objects.," Applied optics 31(29), 6247-54 (1992).

[15] Sharpe, J., Ahlgren, U., Perry, P., Hill, B., Ross, A., Hecksher-sørensen, J., Baldock, R., and Davidson, D., "Optical Projection Tomography as a Tool for 3D Microscopy and Gene Expression Studies," Science 296(5567), 541-545 (2002).

[16] Lorbeer, R., Heidrich, M., Lorbeer, C., Fernando, D., Ojeda, R., Bicker, G., Meyer, H., Heisterkamp, A., Zentrum, L., et al., "Highly efficient 3D fluorescence microscopy with a scanning laser optical tomograph," Optics express 19(6), 412-417 (2011).

[17] Kellner, M., Heidrich, M., Beigel, R., Lorbeer, R.-A., Knudsen, L., Ripken, T., Heisterkamp, A., Meyer, H., Kühnel, M.P., et al., "Imaging of the mouse lung with scanning laser optical tomography (SLOT)," Journal of applied physiology (Bethesda, Md. : 1985) 113(6), 975-83 (2012).

[18] Tinne, N., Antonopoulos, G.C., Mohebbi, S., Andrade, J., Nolte, L., Meyer, H., Heisterkamp, A., Majdani, O., and Ripken, T., "Three-dimensional hard and soft tissue imaging of the human cochlea by scanning laser optical tomography (SLOT)," PLOS ONE 12(9), e0184069 (2017).

[19] Nolte, L., Tinne, N., Schulze, J., Heinemann, D., Antonopoulos, C., Meyer, H., Nothwang, H.G., Lenarz, T., Heisterkamp, A., et al., "Scanning laser optical tomography for in toto imaging of the murine cochlea," PloS one1-10 (2017).

[20] Heidrich, M., Kühnel, M.P., Kellner, M., Lorbeer, R.A., Lange, T., Winkel, A., Stiesch, M., Meyer, H., and Heisterkamp, A., "3D imaging of biofilms on implants by detection of scattered light with a scanning laser optical tomograph,” Biomedical Optics Express 2(11), 2982-2994 (2011).

[21] Boyd, R.W., [Nonlinear optics], Academic Press (2008).

[22] Su, P., Chen, W., Chen, Y., and Dong, C., "Determination of Collagen Nanostructure from Second-Order Susceptibility Tensor Analysis," Biophysj 100(8), 2053-2062 (2011).

[23] Plotnikov, S. V, Millard, A.C., Campagnola, P.J., and Mohler, W.A., "Characterization of the myosin-based source for second-harmonic generation from muscle sarcomeres.," Biophysical journal 90(2), 693-703 (2006).

[24] Andor Technology, "Spectral Imaging," 2018, <http://www.andor.com/learning-academy/spectral-imaging-anoverview-of-andor's-solutions-for-spectral-imaging $>$.

[25] Schneider, C. a, Rasband, W.S., and Eliceiri, K.W., "NIH Image to ImageJ: 25 years of image analysis," Nature Methods 9(7), 671-675 (2012).

[26] Roth, S., Freund, I., and IUCr, "Second harmonic generation and orientational order in connective tissue: a mosaic model for fibril orientational ordering in rat-tail tendon," Journal of Applied Crystallography 15(1), 7278 (1982). 